\title{
Effects of creative expression therapy for older adults with mild cognitive impairment at risk of Alzheimer's disease: a randomized controlled
} clinical trial

This article was published in the following Dove Press journal:

Clinical Interventions in Aging

\author{
Junyu Zhao' \\ Hong $\mathrm{Li}^{1,2}$ \\ Rong Lin' \\ Yuan Wei ${ }^{1}$ \\ Aiping Yang' \\ 'School of Nursing, Fujian Medical \\ University, Fuzhou 350 I08, People's \\ Republic of China; ${ }^{2}$ Department of \\ Nursing, Fujian Provincial Hospital, \\ Fuzhou 35000I, People's Republic \\ of China
}

Correspondence: Hong Li Department of Nursing, Fujian Provincial Hospital, No I34, Dong Street, Gulou Dist, Fuzhou 35000 I, People's Republic of China Tel +86 59l 87557768 Fax +86 59| 22862526 Email leehong99@126.com

\begin{abstract}
Objective: Elderly people with mild cognitive impairment (MCI) are at high risk for dementia. This study compared the effects of standard cognitive training with a creative expression (CrExp) program.

Methods: Adult patients with MCI aged 60 years and older $(\mathrm{N}=93)$ were randomly assigned to either CrExp therapy $(n=48)$ or a control group who received standard cognitive training $(n=45)$ for 16 weeks. The Montreal Cognitive Assessment, Chinese Version of the Auditory Verbal Learning Test, Chinese Version of the Category Verbal Fluency Test, Digit Span Test, Trail Making Test, Chinese Version of Activities of Daily Living scale, and Memory Satisfaction Questionnaire were used to measure cognitive functioning and daily living abilities. Assessments were administered at baseline, postintervention, and 6 months follow-up.

Results: At postintervention, patients receiving CrExp therapy scored significantly higher than patients receiving standard cognitive training, in general cognitive functioning, memory, executive function, functional status, and everyday living ability. The improvements in cognitive functioning were maintained at the 6 month follow-up.

Conclusion: CrExp therapy has greater positive effects on cognitive functions and daily living ability than standard cognitive training. This unique therapy may serve as a cost-effective adjunct to standard interventions for older adults with mild cognitive impairment.
\end{abstract}

Keywords: cognitive impairment, creative expression, non-pharmaceutical therapy, health care

\section{Introduction}

With the world's aging population on the rise, and an increasing prevalence of Alzheimer's disease (AD), dementia has become an important area for global research. Mild cognitive impairment (MCI) is defined as a cognitive disorder associated with deficits in memory, language, executive functioning, attention, and visual-spatial abilities. In Shanghai, China, the prevalence of adult MCI (aMCI) in people aged 60 years and older is $22.3 \% .^{1}$ Research in other cities in China or USA has found a similar prevalence rate..$^{2-4} \mathrm{~A}$ retrospective cohort study found that the progression from MCI to dementia or AD was $\sim 13.5 \%-13.9 \%$ person-years. ${ }^{5}$ The identification and treatment of those at high risk for developing dementia are therefore critical.

The benefits of nonpharmaceutical interventions in older adults with cognitive impairment have been widely reported. ${ }^{6}$ However, a systematic review by Zubala et $\mathrm{al}^{7}$ states that the component of cognitive interventions providing the greatest benefits in older adults is still unclear. Prior research has provided evidence that consistent 
physical training ${ }^{8,9}$ and social activities may maintain neuronal plasticity and increase brain activity in older adults. ${ }^{10}$ Physical exercise has been shown to increase the number of neuronal and non-neuronal cortical cells and hippocampal neuronal cells in adolescent rats. ${ }^{11}$ Other research has shown that specific activities, such as playing sports or an instrument, specifically the drums, provide long-term neuronal adaptation mechanisms and increase visual-spatial abilities. ${ }^{12}$ In addition, animal studies have shown that spatial memory tasks may benefit memory over a 4-month period. ${ }^{13}$

Social behavioral activation (SBA) has been shown to slow cognitive decline in people with MCI or at risk for dementia. ${ }^{14}$ SBA involves components of strength, frequency, duration, and patterns, which have been seen in traditional health care programs. Moreover, tasks involving memory, such as strategy training (creating a weekly plan) and a daily schedule (reading newspaper and sharing to audience) that enhance motivation and demand integration of multiple cognitive pathways, can also lead to a slowing of cognitive decline. ${ }^{15}$

Novel cognitive intervention programs such as Timeslips build upon art therapy and humanities. The storytelling program has been reported in several previous studies, ${ }^{16}$ and it has been suggested that the engaging nature of this group-centered activity may specifically benefit patients emotionally and improve communication skills and memory in older adults with AD or dementia. The details of this creative expression (CrExp) program have been reported in previous publications. ${ }^{17,18}$ Briefly, storytelling is used to strengthen memory in elderly patients with MCI. The CrExp program involves five steps of functional tasks related to the presentation of a picture, including the topic of the picture, the background scenery, the development of a story, the actions of the leading character, and the ending of the story. Symbols, graphs, lines, numbers, and colors can be used to express feelings or emotions in order to narrate a vivid and complete story during the task. The CrExp program is conducted in a group setting.

The "dual-coding theory" of cognition emphasizes that both visual and verbal data are used in memory tasks. This theory suggests that there are two distinct channels for the organization of incoming messages that can be acted upon, stored, and retrieved for subsequent use. ${ }^{19}$ While prior research has shown that storytelling-based CrExp training exerts a positive influence on older adults with $\mathrm{AD}$, whether it can be used as an effective tool in treating elders with MCI or cognitive disorders at risk of dementia remains unclear. The current study explored the effects of a CrExp training program on cognitive functioning in older adults with MCI.
In addition to storytelling, drawing tasks were used as an exercise demanding visual imagery to stimulate parallel cognitive processes and artistic expression.

\section{Methods \\ Study design}

The current study was a 16-week cluster-randomized clinical trial conducted from January 2015 to March 2016 at the outpatient clinic at the Fujian Provincial Hospital. Participants were randomly assigned to either the CrExp group or a control group (CG) who received social activation therapy by computer-generated random numbers. Cluster randomization was carried out by a statistician after screening was completed. Group assignment was concealed until the completion of baseline assessment. The assessor was not involved in the intervention, and the interventionists were not involved in the outcome assessments.

Each participant provided written informed consent, and all procedures were approved by the hospital ethics committee. The study was conducted according to the Declaration of Helsinki and was registered in the Chinese Clinical Trials Registry (trial registration number: ChiCTR-ONN-16007776).

\section{Participants}

Adults aged 60 years or older from the Department of Geriatrics or Neurology at a public tertiary A hospital who complained of memory issues or had suspected cognitive impairment were examined. A total of 93 patients met the following inclusion criteria for $\mathrm{MCI}:{ }^{20}$ memory/cognitive complaint reported by the patient or a caregiver within past year; clinical record of probable MCI according to the Diagnostic and Statistical Manual of Mental Disorders, 4th Edition (DSM-IV); objective cognitive impairment in one or more domains as revealed by neuropsychological assessments; and normal personal selfcare and daily living ability. The exclusion criteria were as follows: known psychiatric cause of cognitive dysfunction; clinical diagnosis of depression or anxiety; history of brain lesion; psychoactive substance abuse; comorbid medical records associated with cognitive functional decline; and taking medications with significant impacts on cognitive function or influence on hearing, vision, or communication that may affect participation in the program or assessments.

\section{Measurements}

Assessments were made by a professional cognitive assessor at baseline, postintervention, and 6 months follow-up. Primary outcomes were the Chinese-Changsha version of 
Montreal Cognitive Assessment (MoCA), ${ }^{21}$ the Chinese Version of the Auditory Verbal Learning Test (CVAVLT), ${ }^{22}$ the Chinese Version of the Category Verbal Fluency Test $(\mathrm{CVCVFT}),{ }^{23}$ the Digital Span Test (DST), ${ }^{24}$ the Chinese version of the Trail Making Test A (TMT-A) and the Trail Making Test B (TMT-B), ${ }^{25}$ and the Chinese Version of Activities of Daily Living scale (CVADL). ${ }^{26}$ The abovementioned scales were used to test the general cognitive function including memory, language, executive, attention, and spatial ability. The Memory Satisfaction Questionnaire (MSQ) was developed to evaluate the affect regarding one's memory by adding up the score of 20 items using a 4-point $(0-4)$ Likert scale. ${ }^{27}$ More information can be found from the reference cited.

\section{Intervention}

\section{CrExp group}

The cognitive program for the CrExp group involved a total of 25 sessions over 16 weeks, facilitated by a group of professional therapists. All sessions began with a 5-10 min warm-up of an interaction game, followed by a $10 \mathrm{~min}$ drawing and then a 30 min core CrExp. Session ended with a 5-10 min discussion and a 5 min conclusion.

\section{CG}

The CG participated in standard cognitive rehabilitation, which involved a total of the same sessions as CrExp group over 16 weeks, facilitated by a group of occupational therapists (social workers, nursing specialists, and physicians). Each session included $10 \mathrm{~min}$ of "finger exercises", ${ }^{28}$ followed by 30 min of core cognitive strategy training (eg, a course, an activity, or a game) and, finally, 5 min of conclusion speeches.

Throughout the study, all participants continued with their routine medical care and out-patient follow-up.

\section{Statistical analysis}

All analyses were performed using STATA 12.0 (StataCorp LP, College Station, TX, USA). ${ }^{29}$ Group differences in demographics and baseline assessments were compared using the appropriate statistical tests (Fisher's exact test, independent samples $t$-test, or chi-square test). The intervention effects, over time from baseline to postintervention and from baseline to 6 months follow-up, were evaluated using repeated measures of ANOVA. The between-group differences at postintervention and 6 months follow-up were performed by ANCOVA with baseline age, education, and MoCA score as covariates. Bonferroni analyses were performed when significant between-group differences were revealed. All data were analyzed according to the intentionto-treat principle. Missing data were replaced by averaging the individual's available data (from first session to last observation). The statistical significance level was set at $P<0.05$ (two-tailed).

\section{Results \\ Participant characteristics}

Of the 134 patients screened for eligibility, 41 patients met some exclusion criteria, 37 patients did not meet inclusion criteria, and four patients declined participation. Therefore, 93 individuals ( 50 males and 43 females), aged 60-85 years (mean $=69.2, \mathrm{SD}=6.3$ ), were randomly assigned to either the CrExp group $(n=48)$ or the CG $(n=45)$. Baseline demographics and a study flow chart are provided in Table 1 and Figure 1, respectively. There were no significant statistical differences in characteristics (range $P=0.253-0.987$ ) or neuropsychological assessment results (range $P=0.339-0.876$ ) between the groups at baseline.

\section{Compliance}

Of the 93 participants who completed the baseline assessment, 88 (94.6\%) participants performed the postintervention evaluation and $84(90.3 \%)$ participants completed the 6 months follow-up (Figure 1). There were no significant differences between compliance and noncompliance participants at postintervention $\left(\chi^{2}[1]=0.876, P=0.349\right)$ or at 6 months follow-up $\left(\chi^{2}[1]=0.376, P=0.540\right)$. Adverse events were not reported from either group.

\section{Effects of Intervention}

All mean group assessment scores and between-group comparisons are illustrated in Table 2 . The results of the repeatedmeasures ANOVA showed significant improvements in the CrExp group in all measurements at postintervention and at 6 months follow-up. Similarly, there was significant improvement in all assessments in the CG except activities of daily living (ADL) and complex social functioning.

At postintervention, the CrExp group showed significantly greater improvement than $\mathrm{CG}$ in general cognitive functions (MoCA; $F=21.47, P<0.001$ ), memory (CVAVLT immediate recall; $F=4.81, P=0.023$ and delay recall; $F=3.98$, $P=0.012$ ), language function (CVCVFT; $F=3.91, P=0.017$ ), executive function (DST; $F=23.35, P<0.001$ ), attention functioning (TMT-B; $F=3.29, P=0.030$ ), and memory satisfaction (MSQ; $F=18.39, P<0.001$ ). When gender and ambulatory level were entered as covariates, there was also 
Table I Baseline demographic and neuropsychological evaluations of participants

\begin{tabular}{|c|c|c|c|}
\hline Characteristics & CrExp group $(n=48)$ & CG $(n=45)$ & $P$-value \\
\hline Age (years), range/mean (SD) & $60-85 / 70.6(6.9)$ & $60-85 / 69.5(6.7)$ & $0.816^{\mathrm{a}}$ \\
\hline Gender, n (\%), female/male & $25(52.1) / 23(47.9)$ & $23(5 \mathrm{I} . \mathrm{I}) / 22(48.9)$ & $0.253^{c}$ \\
\hline Education level, n (\%), illiterate/primary/secondary/tertiary & $3(6.2) / 9(18.8) / 20(4 \mid .7) / 16(33.3)$ & $3(6.7) / 10(22.2) / 19(42.2) / 13(28.9)$ & $0.482^{\mathrm{b}}$ \\
\hline Social status, $n$ (\%), living with family/alone & $47(98.0) / /(0.2)$ & $43(95.6) / 2(4 / 4)$ & $0.503^{c}$ \\
\hline Exercise per day, $\mathrm{n}(\%), 0 /<30 \mathrm{~min} />30 \mathrm{~min}$ & $9(18.8) / 16(33.3) / 23(47.9)$ & $10(22.2) / 14(31.1) / 21(46.7)$ & $0.968^{\mathrm{b}}$ \\
\hline Ambulatory level, $n$ (\%), unaided/with stick & $45(93.8) / 3(6.2)$ & $42(93.3) / 3(6.7)$ & $0.417^{c}$ \\
\hline Medical history, n (\%), hypertension/diabetes/dyslipidemia & $16(33.3) / 24(50.0) / 8(16.7)$ & $15(33.3) / 22(48.9) / 8(17.8)$ & $0.987^{\mathrm{b}}$ \\
\hline MoCA score, mean (SD) & $22.04(2.73)$ & $21.88(2.96)$ & $0.339^{\mathrm{a}}$ \\
\hline NCSE, composite score, mean (SD) & $50.87(11.75)$ & $52.64(12.03)$ & $0.890^{\mathrm{a}}$ \\
\hline NCSE, normal domain, mean (SD) & $4.87(1.75)$ & $5.04(2.13)$ & $0.277^{\mathrm{a}}$ \\
\hline CVAVLT totally recall score, mean (SD) & $14.72(3.47)$ & $15.03(3.16)$ & $0.676^{\mathrm{a}}$ \\
\hline CVAVLT delay recall score, mean (SD) & $5.72(3.21)$ & $5.03(3.16)$ & $0.676^{\mathrm{a}}$ \\
\hline CVCVFT score, mean (SD) & $10.18(1.90)$ & $9.3(2.35)$ & $0.35 I^{\mathrm{a}}$ \\
\hline DST score mean (SD) & $7.34(1.28)$ & $7.45(1.59)$ & $0.876^{\mathrm{a}}$ \\
\hline TMT-A score mean (SD) & $136.29(31.31)$ & $132.14(32.85)$ & $0.605^{a}$ \\
\hline TMT-B score mean (SD) & 238.34 (12.99) & 245.14 (II.59) & $0.763^{\mathrm{a}}$ \\
\hline CVADL score, mean (SD) & $25.49(2.39)$ & $26.06(2.04)$ & $0.407^{\mathrm{a}}$ \\
\hline MSQ score, mean (SD) & $48.13(0.84)$ & $47.26(0.69)$ & $0.633^{\mathrm{a}}$ \\
\hline
\end{tabular}

Notes: andependent sample $t$-test. ' $F$ Fisher's exact test. ' $C$ hi-square test.

Abbreviations: CG, control group; CrExp, creative expression; CVADL, Chinese Version of Activities of Daily Living; CVAVLT, Chinese Version of the Auditory Verbal Learning Test; CVCVFT, Chinese Version of the Category Verbal Fluency Test; DST, Digital Span Test; MoCA, Montreal Cognitive Assessment; MSQ, Memory Satisfaction Questionnaire; NCSE, Neurobehavioral Cognitive Status Examination; TMT-A, Trail Making Test A; TMT-B, Trail Making Test B.

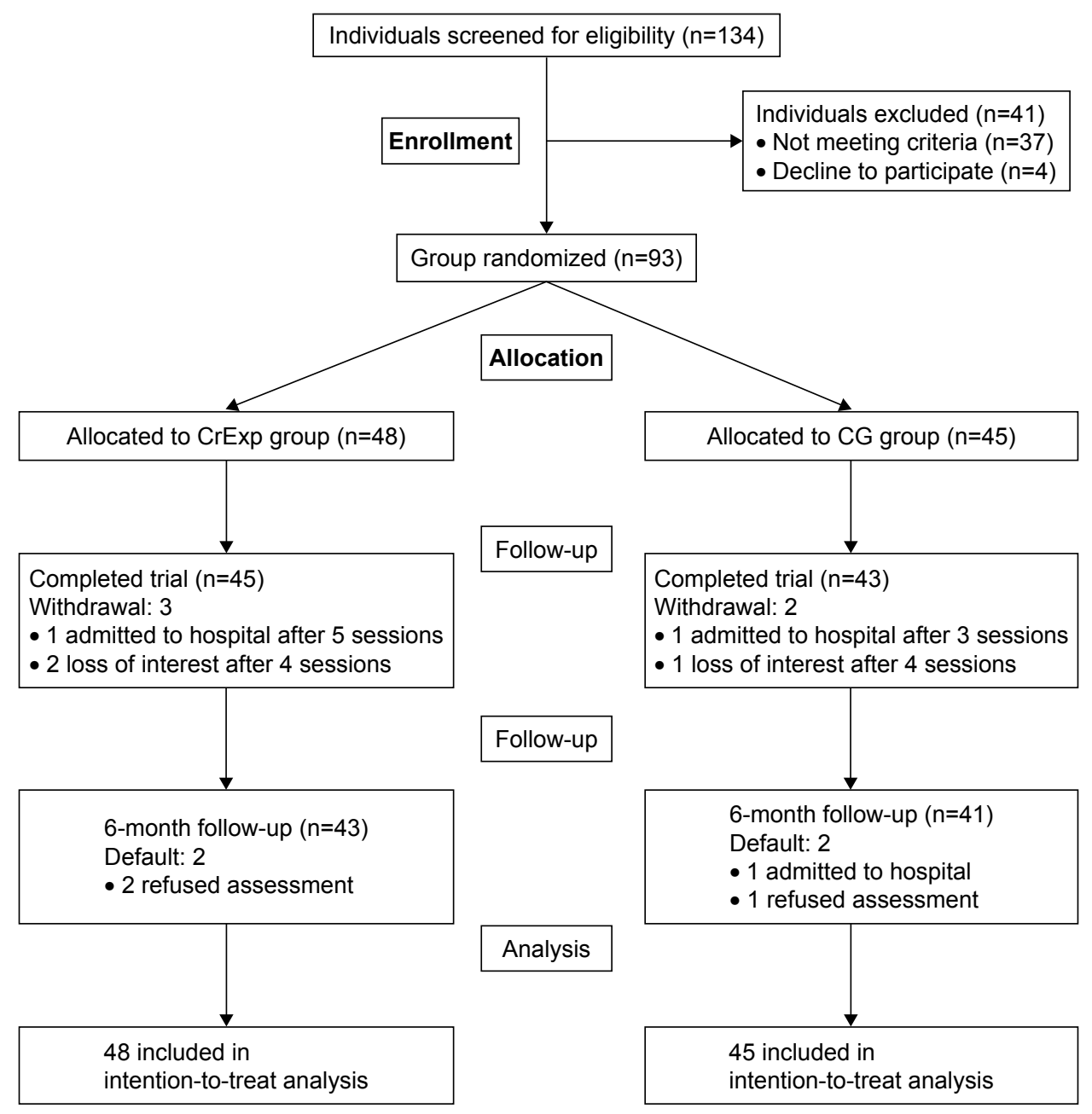

Figure I Study flow chart.

Abbreviations: CG, control group; CrExp, creative expressions group. 


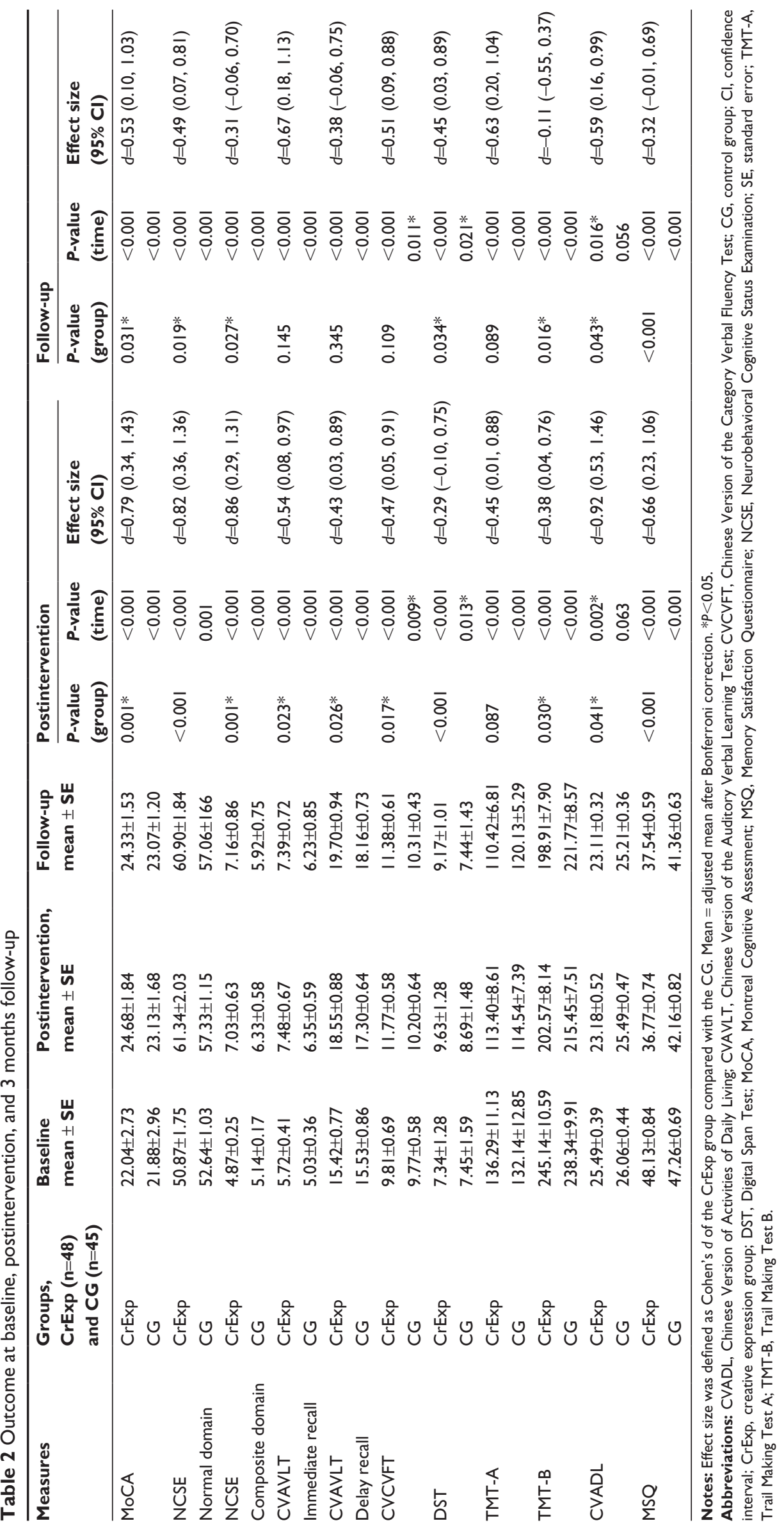


a significant difference between groups in ADL (CVADL; $F=2.78, P=0.041)$.

\section{Sustainability of effects}

At 6 months follow-up, significant group differences remained in general cognitive functioning (MoCA; $F=4.89$, $P=0.031$ ), memory (CVAVLT delay recall; $F=5.14$, $P=0.025$ ), executive function (DST; $F=4.35, P=0.034$ ), attention functioning (TMT-B; $F=6.19, P=0.016$ ), ADL (CVADL; $F=4.24, P=0.043$ ), and memory satisfaction (MSQ; $F=7.49$, $P=0.008)$.

\section{Discussion}

The aim of this study was to compare the effectiveness of a CrExp training program with standard cognitive therapy in older adults with $\mathrm{MCI}$ at risk of dementia. The participants in the CrExp group showed greater improvements in general cognitive functioning, memory, executive functioning, attention, and ADL compared with the CG both at postintervention and at 6 months follow-up.

The results of the current study concur with previous studies showing that strengthening and demanding tasks, along with various behavioral exercises, lead to greater improvement in cognitive functioning than either treatment alone. ${ }^{30,31}$ While physical exercise and cognitive training were involved in the CG treatment, these interventions are likely practiced at home and, thus, did not provide further enhancement. The process of integrating professional resources and intensive training guidance, on the other hand, may increase cognitive motivation. However, there is a weak evidence for improvement in the domains of memory and executive function after short-term cognitive intervention for older adults with MCI and, as such, long-term intervention (as provided in the current study) may be critical.

Participants in the CrExp group showed significantly greater improvement than those in the CG, in general cognitive function, especially in executive functioning, language, and attention. These results strengthen the argument for the necessity of more frequent and longer sessions of CrExp programs in MCI patients. In addition, cognitive therapy should focus on consistent creative exercises involving imagination and narration.

While ADL was significantly improved at postintervention and at 6 months follow-up in the CrExp group, there was no significant improvement in CG. The ADL measures the degree of impairment in everyday basic functioning, evaluates the prognosis, and predicts daily functioning and motor performance in diseases associated with neurocognitive disorders or insufficient mental activity. ${ }^{32,33}$ ADL is intimately linked to working memory and executive and visuospatial functioning, which can be facilitated through sustained cognitive training. ${ }^{34}$ The significant improvement in ADL performance in the CrExp group may be attributed to an enhancement in working memory through the practice of imaging, storytelling, and drawing exercises. More studies are needed to explore factors that correlate with improved ADL.

To our knowledge, the present study is the first to report on CrExp intervention in China. Patients with MCI may not present with significant neuropsychological deficits, but instead with mild cognitive dysfunction, especially in memory, speed, and accuracy, which have been associated with the normal aging process..$^{35}$ During CrExp exercises, MCI patients are given the opportunity to use creativity, imagination, and thinking processes that may otherwise remain used. In addition, those who participate in CrExp share stories with peers who may naturally stimulate improved communication, attention, and expression abilities as well as general cognition and well-being.

\section{Limitations}

While the present study provides evidence for the beneficial use of CrExp intervention in the elderly, there are limitations that warrant mentioning. First, the current study had a relatively small sample size that may not reliably represent MCI patients across clinical subtypes. In addition, participants were all residents of Fuzhou, China, and as such, the results may not generalize to other regions of China or other countries. Finally, due to limited human resources and shortterm follow-up period, future studies should be designed to follow participants for at least 1 year postintervention.

\section{Conclusion}

The current randomized controlled trial provides evidence that a CrExp program involving storytelling and picturedrawing tasks is effective in improving cognitive functioning and ADL in older adults at risk for dementia. In addition, the results suggest that cognitive improvements are sustained for at least 6 months postintervention. Health care providers should consider CrExp interventions as part of the treatment program for older adults with MCI.

\section{Acknowledgments}

We thank Dr MingPing Ma and Dr XiaoDong Pan for their helpful contributions to this project; Qiuhua Chen and Jiawei Su in Fujian Provincial Hospital for the use of 
facilities and contributing to perform the examination and recruitment; and Tingting Wu, Jing Yan, and Huiying Chen for assistance with training and program follow-up. This research is supported by National Nature Science Foundation of China (81571379).

\section{Disclosure}

The authors report no conflicts of interest in this work.

\section{References}

1. Wang T, Xiao S, Chen K, et al. Prevalence, incidence, risk and protective factors of amnestic mild cognitive impairment in the elderly in Shanghai. Curr Alzheimer Res. 2017;14(4):460-466.

2. Su X, Shang L, Xu Q, et al. Prevalence and predictors of mild cognitive impairment in Xi'an: a community-based study among the elders. PLoS One. 2014;9(1):e83217.

3. Keyimu K, Zhou X-H, Miao H-J, Zou T. Mild cognitive impairment risk factor survey of the Xinjiang Uyghur and Han elderly. Int J Clin Exp Med. 2015;8(8):13891-13900.

4. Pankratz VS, Roberts RO, Mielke MM, et al. Predicting the risk of mild cognitive impairment in the Mayo Clinic Study of Aging. Neurology. 2015;84(14):1433-1442.

5. Tifratene K, Robert P, Metelkina A, Pradier C, Dartigues JF. Progression of mild cognitive impairment to dementia due to $\mathrm{AD}$ in clinical settings. Neurology. 2015;85(4):331-338.

6. Hilfiker R, Meichtry A, Eicher M, et al. Exercise and other nonpharmaceutical interventions for cancer-related fatigue in patients during or after cancer treatment: a systematic review incorporating an indirect-comparisons meta-analysis. Br J Sports Med. 2018;52(10): 651-658.

7. Zubala A, Macgillivray S, Frost H, et al. Promotion of physical activity interventions for community dwelling older adults: a systematic review of reviews. PLoS One. 2017;12(7):e0180902.

8. Hauer K, Ullrich P, Dutzi I, et al. Effects of standardized home training in patients with cognitive impairment following geriatric rehabilitation: a randomized controlled pilot study. Gerontology. 2017; 63(6):495-506.

9. Hagovska M, Nagyova I. The transfer of skills from cognitive and physical training to activities of daily living: a randomised controlled study. Eur J Ageing. 2017;14(2):133-142.

10. Shimada H, Ishii K, Makizako H, Ishiwata K, Oda K, Suzukawa M. Effects of exercise on brain activity during walking in older adults: a randomized controlled trial. $J$ Neuroeng Rehabil. 2017;14(1):50.

11. Victorino AB, Serra FT, Piñero PP, et al. Aerobic exercise in adolescence results in an increase of neuronal and non-neuronal cells and in mTOR overexpression in the cerebral cortex of rats. Neuroscience. 2017;361:108-115.

12. Bianco V, Berchicci M, Perri RL, Quinzi F, Di Russo F. Exerciserelated cognitive effects on sensory-motor control in athletes and drummers compared to non-athletes and other musicians. Neuroscience. 2017;360:39-47.

13. Nonaka M, Fitzpatrick R, Lapira J, et al. Everyday memory: towards a translationally effective method of modelling the encoding, forgetting and enhancement of memory. Eur J Neurosci. 2017;46(4): 1937-1953.

14. Henry JD, von Hippel W, Thompson C, Pulford P, Sachdev P, Brodaty H. Social behavior in mild cognitive impairment and early dementia. J Clin Exp Neuropsychol. 2012;34(8):806-813.

15. Rodakowski J, Reynolds CF, Lopez OL, Butters MA, Dew MA, Skidmore ER. Developing a non-pharmacological intervention for individuals with mild cognitive impairment. J Appl Gerontol. 2018; 37(5):665-676.
16. George DR, Houser WS. "I'm a storyteller!": exploring the benefits of TimeSlips creative expression program at a nursing home. Am J Alzheimers Dis Other Demen. 2014;29(8):678-684.

17. Houser WS, George DR, Chinchilli VM. Impact of TimeSlips creative expression program on behavioral symptoms and psychotropic medication use in persons with dementia in long-term care: a clusterrandomized pilot study. Am J Geriatr Psychiatry. 2014;22(4):337-340.

18. Hydén L-C. Storytelling in dementia: embodiment as a resource. Dementia (London). 2013;12(3):359-367.

19. Katz A. Allan Urho Paivio (1925-2016). Am Psychol. 2017;72(5):497.

20. Jia J, Wang Y, Zhang C. Chinese guidelines for diagnosis and management of cognitive impairment and dementia (I): dementia diagnostic procedures. Zhonghua Yi Xue Za Zhi. 2011;91(9):577-581.

21. Tu Q, Jin H, Ding B, et al. Reliability, validity, and optimal cutoff score of the Montreal cognitive assessment (Changsha Version) in ischemic cerebrovascular disease patients of Hunan Province, China. Dement Geriatr Cogn Dis Extra. 2013;3(1):25-36.

22. Zhao Q, Lv Y, Zhou Y, Hong Z, Guo Q. Short-term delayed recall of auditory verbal learning test is equivalent to long-term delayed recall for identifying amnestic mild cognitive impairment. PLoS One. 2012; 7(12):e51157.

23. Zhao Q, Guo Q, Ding D, et al. The effect of age, gender and education in cognitively normal elderly on the strategy of two different category verbal fluency tests. Alzheimers Dementia. 2010;6(4):S22.

24. Lortie JJ, Remington R, Hoffmann H, et al. The WAIS digit span test: a sensitive indicator of cognitive decline in mild cognitive impairment. Gerontologist. 2012;51(4):304.

25. Tombaugh TN. Trail making test A and B: normative data stratified by age and education. Arch Clin Neuropsychol. 2004;19(2):203.

26. Chu TK, Chung JC. Psychometric evaluation of the Chinese version of the activities of daily living questionnaire (ADLQ-CV). Int Psychogeriatr. 2008;20(6):1251.

27. Troyer AK, Rich JB. Psychometric properties of a new metamemory questionnaire for older adults. J Gerontol B Psychol Sci Soc Sci. 2002; 57(1): 19 .

28. Singh T, Zatsiorsky VM, Latash ML. Contrasting effects of fatigue on multifinger coordination in young and older adults. J Appl Physiol. 2013;115(4):456-467.

29. Barquera S, Carrión C, Campos I, et al; Stata Corp. Stata Reference Manual. Release 7. College Station, TX: Stata Press; 2001.

30. Noreen S, MacLeod MD. What do we really know about cognitive inhibition? Task demands and inhibitory effects across a range of memory and behavioural tasks. PLoS One. 2015;10(8):e0134951.

31. Torisson G, Stavenow L, Minthon L, Londos E. Importance and added value of functional impairment to predict mortality: a cohort study in Swedish medical inpatients. BMJ Open. 2017;7(5):e014464.

32. Tomaszewski Farias S, Giovannetti T, Payne BR, et al. Self-perceived difficulties in everyday function precede cognitive decline among older adults in the ACTIVE Study. J Int Neuropsychol Soc. 2018;24(1): 104-112.

33. Oh EY, Jung MS. Effects of a cognitive training program on cognitive function and activities of daily living in patients with acute ischemic stroke. J Korean Acad Nurs. 2017;47(1):1-13.

34. Phillips LJ, Reid-Arndt SA, Pak Y. Effects of a creative expression intervention on emotions, communication, and quality of life in persons with dementia. Nurs Res. 2010;59(6):417-425.

35. Lassen-Greene CL, Steward K, Okonkwo O, et al. Mild cognitive impairment and changes in everyday function over time: the importance of evaluating both speed and accuracy. J Geriatr Psychiatry Neurol. 2017;30(4):220-227. 


\section{Publish your work in this journal}

Clinical Interventions in Aging is an international, peer-reviewed journal focusing on evidence-based reports on the value or lack thereof of treatments intended to prevent or delay the onset of maladaptive correlates of aging in human beings. This journal is indexed on PubMed Central, MedLine,

CAS, Scopus and the Elsevier Bibliographic databases. The manuscript management system is completely online and includes a very quick and fair peer-review system, which is all easy to use. Visit http://www.dovepress. com/testimonials.php to read real quotes from published authors. 